\title{
Comparación entre Soluciones Numéricas de un Modelo Matemático de Combustión Teórica y Variación de la Conductividad del Cuesco de Palma en un Reactor de Lecho Fijo
}

\author{
Luz M. Ahumada, Cinthia P. Audivet, Antonio J. Bula y Marco E. Sanjuan \\ Facultad de Ingeniería, Dpto. de Ingeniería Mecánica, Univ. del Norte, Barranquilla-Colombia \\ (e-mail: Imahumada@Uninorte.edu.co; caudivet@uninorte.edu.co; abula@uninorte.edu.co; \\ msanjuan@uninorte.edu.co)
}

Recibido Nov. 22, 2016; Aceptado Ene. 10, 2017; Versión final Mar. 8, 2017, Publicado Jun. 2017

\begin{abstract}
Resumen
En esta investigación se llevó a cabo la comparación entre diferentes soluciones numéricas para un modelo matemático de combustión teórica y la variación de la conductividad del cuesco de palma en un reactor de lecho fijo descendente mediante combinaciones entre los esquemas compactos (EC) de diferencias finitas (DF) de orden 2, 4 y 6 y los métodos de integración de Runge Kutta (RK) para $n=1,2$ y 4. Los resultados obtenidos mostraron que la aproximación obtenida bajo el EC y el método de integración $\mathrm{RK}$, ambos de orden 4, fué la más cercana al punto de referencia analizado (EC de orden $\mathrm{h}^{6}$, sistema integrador RK de orden 4, malla de $80 \times 80$ y paso de tiempo de 1 segundo) con diferencias del orden de $13.6 \mathrm{E}-05 \%$ y errores porcentuales más significativos entre $7.22 \%$ y $8.1 \%$ a una altura aproximada de $0.5 \mathrm{~m}$ medida desde el fondo de la tolva. Además, se muestra la correlación entre las variables posición-temperatura y temperatura-conductividad para 21 puntos escogidos $x$-espaciados distribuidos sobre una línea que va desde $0 \leq \mathrm{y} \leq \mathrm{H}$. Se corrobora que la conductividad del material aumenta a medida que se incrementa la temperatura y es inversamente proporcional a la distancia medida desde la base menor de la tolva.
\end{abstract}

Palabras clave: esquemas compactos; diferencias finitas; métodos de integración; biomasa; conductividad térmica

\section{Comparison of Numerical Solutions for a Mathematical Model of Theoretical Combustion and Conductivity Variation of Shell Palm in an Fixed Bed Reactor}

\begin{abstract}
In this investigation numerical solutions for a mathematical model of theoretical combustion and conductivity variation of the shell palm in an downdraft fixed bed reactor were compared by means of combinations between order 2, 4 and 6 of finite difference (FD) compact schemes (CE) and Runge Kutta (RK) integration methods ( $n=1,2$ and 4$)$. The results obtained showed that the approximation obtained under 4 order CE and the integration method $\mathrm{RK}(\mathrm{n}=4)$, was the closest with $13.6 \mathrm{E}-5 \%$ to the reference point (order 6 of $\mathrm{CE}$, integrating system RK of order 4, mesh $80 \times 80$ and 1 second step time) and between $7.22 \%$ and $8.1 \%$ significant errors at an approximate height of $0.5 \mathrm{~m}$ measured from the bottom hopper. In addition, the correlation between the position-temperature and temperature-conductivity variables for $21 \mathrm{x}$-spaced points distributed over a line from $0 \leq \mathrm{y} \leq \mathrm{H}$ is presented. This corroborates the fact that the conductivity of the material increases as the temperature increases and is inversely proportional to the distance measured from the bottom hopper.
\end{abstract}

Keywords: compact schemes; finite difference; integration methods; biomass; thermal conductivity 


\section{INTRODUCCIÓN}

Actualmente el uso de biomasa como una fuente de energía amigable con el medio ambiente ha causado mucho interés alrededor del mundo, como evidencia de ello se tiene que la biomasa contribuye aproximadamente entre $10 \%$ y $14 \%$ del suministro de energía a nivel mundial como lo mencionó en su trabajo Ahmad et al. (2016). Ahora bien, El proceso de conversión de la biomasa en energía útil comprende cuatro etapas referenciadas en el trabajo de Kumar et al. (2009): Preprocesamiento de la biomasa, conversión termoquímica, limpieza del gas de síntesis y aprovechamiento del gas producido, como se muestra en la figura 1. Considerando para esto claro está, la adaptación de una cadena de suministro que permita atender el acopio de los residuos generados y que garantice la disponibilidad de las cantidades requeridas en el proceso productivo como lo expuso García-Ubaque et al. (2013).

La conversión termoquímica se convierte en el centro de todo el proceso, ya que de sus condiciones de operación dependerá la composición del gas, cantidad de impurezas y la eficiencia en la conversión de energía, a raíz de ello ha sido motivo de estudio en las últimas décadas.

La optimización de las condiciones del proceso de conversión termoquímica, el aprovechamiento del potencial energético de la biomasa y la construcción de modelos matemáticos que describan su dinámica han motivado a los investigadores a utilizar diferentes técnicas de aproximación y/o experimentales. En la literatura se reporta desde el empleo de metodologías experimentales (Moreno et al., 2009; Melgar et al., 2009; Mehdi et al., 2015; Yepez et al., 2012; Ahumada et al., 2016) como el trabajo desarrollado por Ahumada et al. (2016) de estudios experimentales realizados para un proceso de microgasificación a fin de encontrar correlaciones entre variables que afectaran el poder calorífico del gas de síntesis obtenido del proceso, desarrollo de modelos de transferencia de calor y exergéticos (Mendoza et al., 2012), planteamientos teóricos desarrollados para estudiar un proceso específico de conversión termoquímica (Perez et al., 2010), como es el caso de la gasificación de biomasa mediante la aplicación de técnicas de optimización y modelos de predicción por redes neuronales basados en datos del proceso propiamente dicho desarrollado por Obviedo et al. (2014), por ejemplo, hasta simulaciones numéricas como una aproximación al fenómeno (Chen et al., 2000; Chen et al., 2001; Sordi et al., 2009; Qingluan et al., 2015), y es quizá esta ultima la herramienta más utilizada después de las metodologías experimentales.

Específicamente en el tema de resolución de modelos de transferencia de calor mediante esquemas compactos de diferencias finitas y los métodos de integración de Runge Kutta se reportan en la literatura pocos trabajos, entre esos el desarrollado por Sekhar et. al (2016), quienes obtuvieron buenas aproximaciones para los números de Grashof en un intervalo de 0.05-125 y Prandtl de 0.72-7 resolviendo las ecuaciones de Navier-Stokes y energía usando esquemas compactos de orden superior en coordenadas polares para la transferencia de calor convectiva laminar de una esfera sólida, o el modelo de transferencia de calor 3D micro generalizado en un sistema N-carrier desarrollado por Di Zhao y Weizhong (2010), en el cual se muestran tasas de convergencia alrededor de 2.0 con respecto a la variable espacial $r$ mediante esquemas compactos de segundo orden, lo que mejora la precisión del esquema de Crank-Nicholson acoplado a la aproximación convencional de primer orden para la condición de frontera de Neumann. Además de esto, existen muy pocos trabajos en los cuales se aborden procesos termoquímicos, teniendo en cuenta la variación de la conductividad de la biomasa como es en este caso, con el fin de poder obtener el perfil de temperaturas al interior de la tolva en donde se da inicio al proceso de calentamiento de la biomasa previo a la entrada al reactor.

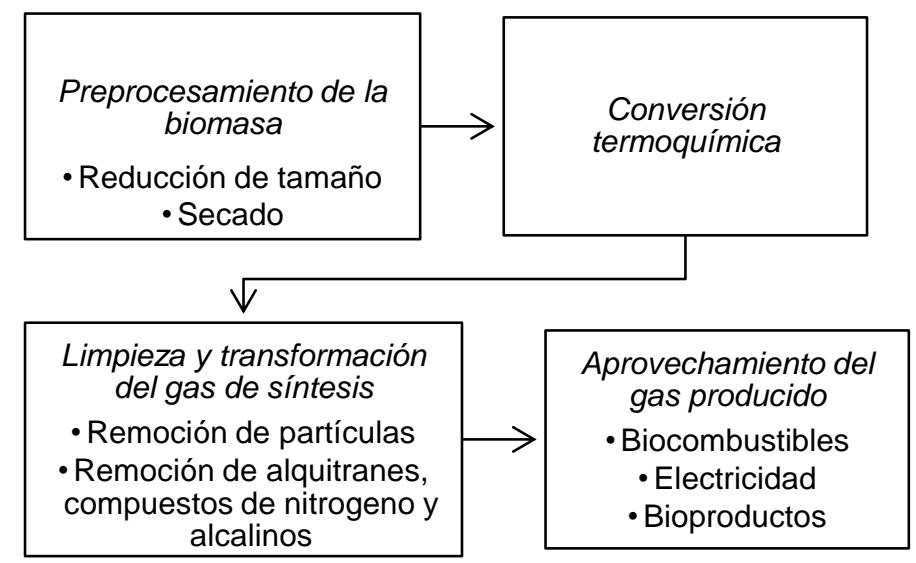

Fig. 1: Proceso de conversión de la biomasa en energía útil 
Como ya se mencionó, los sistemas de aproximación numérica por diferencias finitas y métodos de integración para la resolución fenómenos de transferencia de calor o que incluyan modelos y parámetros físicos de un proceso propiamente dicho, no son comúnmente utilizados. A raíz de ello, esta investigación tuvo como objetivo la simulación de las variables: temperatura y conductividad de la biomasa cuesco de palma africana en función de la posición en la tolva de un reactor de lecho fijo para un proceso de combustión teórica, considerando parámetros propios de la biomasa como el análisis último en base seca del material, a través de la aplicación de técnicas de esquemas compactos (E.C.) de diferencias finitas (D.F.) y métodos de integración de Runge Kutta. Los resultados presentan la comparación entre la precisión de dos métodos de aproximación combinados con tres métodos de integración: diferencias finitas por esquemas compactos de segundo orden - Euler explícito - Runge Kutta de orden 2 - Runge Kutta de orden 4 y diferencias finitas por esquemas compactos de cuarto orden - Euler explícito - Runge Kutta de orden 2 - Runge Kutta de orden 4. Y finalmente, el punto de referencia para realizar la comparación de la precisión de los métodos correspondió a la simulación del modelo basado en el uso de diferencias finitas con esquemas compactos de sexto orden - Runge Kutta de cuarto orden.

\section{FÍSICA DEL PROCESO}

Para establecer el modelo físico que describiera el fenómeno de transferencia de calor en el reactor se consideró un proceso con combustión estequiométrica y condiciones teóricas de frontera e iniciales referentes a temperaturas. El reactor objeto de estudio de este trabajo es un gasificador de lecho fijo descendente donde la biomasa va cayendo progresivamente al lecho y el aire (agente gasificante) entra directamente a la zona de combustión. El esquema considerado en esta investigación se muestra en la Figura 2, se trata de un reactor de corriente descendente construido por la empresa Ankur Scientific modelo WBG - 20 in Scrubbed y este modelo se escogió a consideración del autor debido a la disponibilidad de los datos técnicos del equipo.

Consta de una tolva de forma cónica que termina en una garganta con un estrecho de $120 \mathrm{~mm}$ de diámetro y un ángulo de 45 grados, donde se mezcla la biomasa con el aire, y esta misma finaliza sobre un lecho de carbón que recibe las cenizas del proceso. El flujo de aire que entra al reactor se controla estrangulando el flujo de agua de la bomba conectada al eyector que genera la succión de los gases combustibles por efecto Venturi y una válvula de compuerta permite regular el caudal de agua que arrastra los gases. De esta manera, la transferencia de calor es ascendente tomando como punto inicial el lecho y va disminuyendo a medida que recorre la tolva hasta llegar al tope; se estableció entonces, una ecuación a través de la cual se conoce en cualquier punto de la tolva el valor de la temperatura y la conductividad del material (valores teóricos).
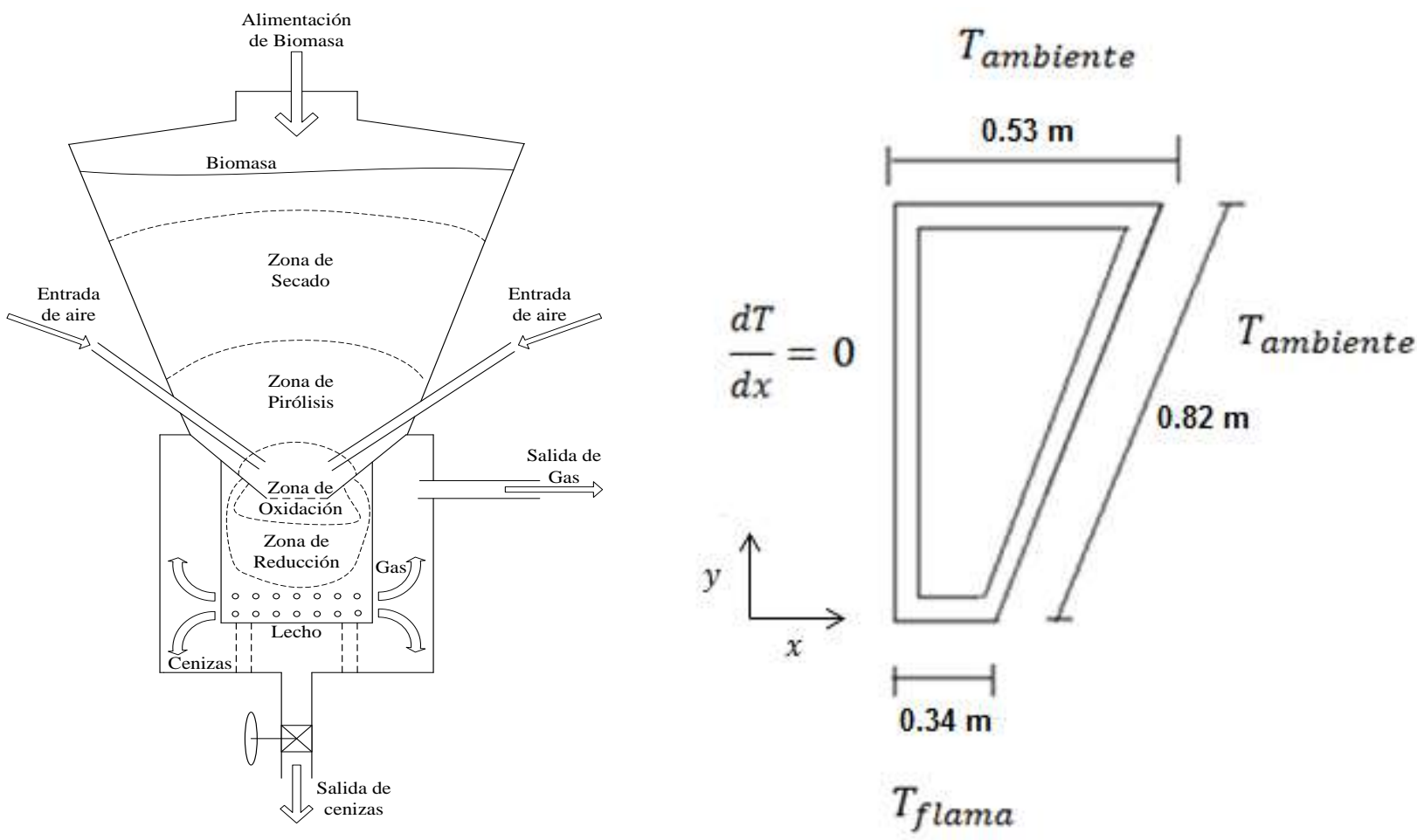

Fig. 2: (a) Esquema del proceso/Tolva, (b) Fenómeno considerado 
La biomasa considerada para la evaluación del proceso en este documento fue el cuesco de palma africana con granulometría promedio de $4.86 \mathrm{~mm}$ y los valores de referencia empleados provienen de bibliografía citada para estos mismos, como es el caso del análisis último de la biomasa en base seca resumido en la Tabla 4 y los valores de las entalpías de formación y masas molares de algunas sustancias como carbón, nitrógeno y agua resumidos en la Tabla 3, empleados para la determinación de la temperatura de flama del proceso. Por otro lado, el constituyente principal de una biomasa vegetal sólida de alta densidad es la celulosa, principal compuesto estructural de las paredes celulares y del tejido vegetal en general. Por lo tanto para madera común y la mayoría de las biomasas compactas se considera una densidad entre los $1500-1530 \mathrm{~kg} / \mathrm{m}^{3}$ (Basu, 2010; Ninduangdee y kuprianov, 2013); por lo cual, en este trabajo se considera una densidad promedio al rango mencionado. Ahora bien, respecto al calor específico de la biomasa, se sabe que esta variable termodinámica varía con la temperatura y la humedad de la especie; sin embargo para facilitar los cálculos en este trabajo se asumió como un valor teórico constante referenciando en la gráfica de la variación del calor específico para la madera, corteza y cenizas de la misma del trabajo desarrollado por Gupta et al. (2003) y tomando el valor máximo promedio de calor específico alcanzado considerando la curva de la madera y de la corteza de la misma especie.

A través de la metodología que a continuación se presenta, considerando 2 modelos matemáticos teóricos (ecuación de difusión y ecuación de la variación de la conductividad de la biomasa en función de la temperatura) y la resolución por esquemas compactos de diferencias finitas de orden 2, 4 y 6 y los sistemas de integración de Runge Kutta para $n=1,2$ y 4, se comparan los resultados obtenidos de las simulaciones para las combinaciones de estos 2 últimos métodos numéricos a fin de correlacionar las variables posición-temperatura y temperatura-conductividad de la biomasa al interior de la tolva del reactor a través un gráfico de superficie de respuesta.

\section{METODOLOGÍA}

Los modelos presentados en este trabajo se basan en estimaciones teóricas y se limitan a condiciones específicas como la homogenización del material de alimentación al proceso (biomasa). Además de esto, dichos modelos matemáticos corresponden a una adaptación para las condiciones consideradas en este trabajo referenciando en algunas ecuaciones desarrolladas por otros autores. La escogencia de estos modelos fue animada por la simplicidad de los mismos a la hora de resolverlos por esquemas compactos y los sistemas de integración de Runge Kutta y debido a las limitaciones para tomar datos por experimentación de las variables de interés correspondientes a la temperatura y conductividad del material respecto a una posición determinada al interior de la tolva. Como condiciones de frontera, en el fondo de la tolva se asumió la temperatura de flama adiabática en el reactor como un valor aproximado (teórico) de la temperatura en esta zona; por otro lado, la temperatura en el tope de la tolva se estableció considerando en este punto una frontera de radiación y resolviendo el sistema para estado estable (temperatura ambiente, aguas arriba), y finalmente, para la condición inicial $\mathrm{t}=0$ del problema se asumió una temperatura ambiente.

Inicialmente, se establecieron los modelos matemáticos para la obtención de la variación de la conductividad del material al interior de la tolva del reactor respecto a una posición especificada en este mediante las ecuaciones (1) y (2). En la primera ecuación se obtiene la resolución en coordenadas cartesianas de la variación de la temperatura respecto a una posición mediante un balance de energía para el volumen de control que se muestra en la Figura 2 (b) o la llamada ecuación de difusión (Ibarra, 2012). Dónde $\rho$ representa la densidad en $\mathrm{kg} / \mathrm{m}^{3}, C p$ la capacidad calorífica en $\mathrm{J} / \mathrm{kgK}$ y $\mathrm{k}$ la conductividad en W/mK de la biomasa, y los 3 términos restantes corresponden a las derivadas parciales de la temperatura de la biomasa respecto a una coordenada espacial y el tiempo. En la ecuación (2) por su parte, se establece la variación de la conductividad del material respecto a la temperatura. De modo que, $\mathrm{k}$ corresponde a la conductividad de la biomasa en $\mathrm{W} / \mathrm{mK}$, a y $\mathrm{b}$ son unas constantes y $\mathrm{T}$ corresponde a la temperatura de la misma biomasa en $\mathrm{K}$, y este ecuación se constituyó basandose en un modelo de ajuste para las curvas de conductividad térmica adoptadas para la madera en el trabajo desarrollado por Moura et al., (2007).

Seguidamente, se determinan los valores de las constantes a y b de la ecuación (2), la temperatura de flama que se emplea como condicion de frontera en el lecho del reactor y finalmente se llevaron a cabo las simulaciones correspondientes a la resolución del fenómeno mediante esquemas compactos y y los métodos de integración de Runge Kutta.

$$
\rho C p \frac{\partial T}{\partial t}=k\left(\frac{\partial^{2} T}{\partial x^{2}}+\frac{\partial^{2} T}{\partial y^{2}}\right)
$$


En esta ecuación

$$
k=a+b T \text { y } \rho=1515 \mathrm{~kg} / \mathrm{m}^{3} \quad y \quad C_{p}=1760 \mathrm{~J} / \mathrm{kgK}
$$

En la Tabla 1 por su parte, se resumen las condiciones de frontera e iniciales mencionadas con anterioridad y que corresponden a las consideraciones de los modelos propuestos en este trabajo y esquematizados en la Figura 2; donde, $x$ representa la dirección horizontal de transferencia de calor, $y$ representa la dirección vertical de transferencia de calor, T flama Representa la temperatura de flama adiabática en el fondo de la tolva, Tambiente representa la temperatura ambiente en las zonas especificadas y $t$ alude a la variable tiempo.

Tabla 1: Condiciones de frontera del proceso

$y=0 \rightarrow T=T$ flama $=1677.6 \mathrm{~K}$
$y=H \rightarrow T=T$ ambiente $=308 \mathrm{~K}$
$\quad x=0 \rightarrow \frac{d T}{d x}=0$
$x=L \rightarrow T=T$ ambiente $=308 \mathrm{~K}$
$t=0 \rightarrow T=T$ ambiente $=308 \mathrm{~K}$

En la Tabla (2) se muestran los valores de variación de la conductividad de la madera respecto a la variación de la temperatura. Estos datos fueron adoptados del trabajo desarrollado por Moura et al. (2007) donde la conductividad inicial de $0.20 \mathrm{~W} / \mathrm{m} . \mathrm{K}$, extraída de la curva publicada por Urakami y Fukayama (1981), referenciado por Harada et al. (1998), correlaciona los valores de conductividad térmica a temperatura ambiente con la densidad de la madera. Estos valores fueron empleados mediante una aproximación lineal para determinar las constantes a y b (0.1280 y 0.0002 respectivamente) que se emplearon en este trabajo para completar el desarrollo del modelo. Luego entonces, el uso de estos datos no es mas que una aproximación para determinar los valores de las constantes de interés dadas las similitudes entre materiales (biomasas vegetales).

Tabla 2: Datos utilizados para calcular a y b (Moura et al., 2007)

\begin{tabular}{cc}
\hline Temperatura $(\mathrm{K})$ & Conductividad $(\mathrm{W} / \mathrm{mK})$ \\
\hline 273 & 0.20 \\
373 & 0.20 \\
473 & 0.20 \\
573 & 0.20 \\
673 & 0.20 \\
773 & 0.23 \\
873 & 0.25 \\
973 & 0.27 \\
1073 & 0.30 \\
1173 & 0.32 \\
1273 & 0.35 \\
\hline
\end{tabular}

El modelo que se consideró en este documento para el cálculo de la temperatura de flama fué basado en el equilibrio químico de las reacciones y la composición elemental de la biomasa. La biomasa se consideró como un compuesto de formula $\mathrm{CH}_{x} \mathrm{O}_{y} \mathrm{~N}_{z}$ donde se toma la composición obtenida del análisis último de la biomasa en base seca, para el cálculo de $x, y, z$. De un balance de energía con primera ley para el reactor considerado, asumiendo un flujo estable y condiciones adiabáticas, se detalla la siguiente ecuación (3) aplicada a los componentes considerados en este trabajo como reactivos (biomasa) y productos (Agua y dióxido de carbono), para el cálculo de la temperatura de combustión adiabática asumida como la máxima temperatura alcanzada por las reacciones del proceso (teóricamente).

Donde; $H_{i}^{\circ}=\stackrel{\circ}{h}_{i}+\left(\bar{h}_{T}-\bar{h}_{T 0}\right)_{i}$ representa las diferencias de entalpia a las condiciones alcanzadas por cada componente. 
Tabla 3: Parámetros y valores de las sustancias de interés (Wark, K. y Richards, D., 1999)

\begin{tabular}{ccc}
\hline & $\overline{\Delta h}_{f, 298}^{\circ}$ & $\bar{h}_{T}-\bar{h}_{298}$ \\
Sustancia & $\mathrm{kJ} / \mathrm{kmol}$ & $\mathrm{kJ} / \mathrm{kmol}$ \\
& & \\
$\mathrm{H}_{2} \mathrm{O}$ & -241820 & 9904 \\
$\mathrm{CO}_{2}$ & -393520 & 9364 \\
$\mathrm{C}$ & 0 & - \\
$\mathrm{H}_{2}$ & 0 & 8468 \\
$\mathrm{~N}_{2}$ & 0 & 8669 \\
$\mathrm{O}_{2}$ & 0 & 8682 \\
\hline
\end{tabular}

Para obtener los datos de la biomasa considerada se emplearon las ecuaciones (4) y (5). $H H V_{\text {Biomasa }}$ es el poder calorífico superior de la biomasa en $\mathrm{kJ} / \mathrm{kmol}$ determinado por la ecuación desarrollada por Channiwala y Parikh (2002) y mostrado en la ecuación (4); donde $M_{C H \times O y N z}$ representa la masa molar de la biomasa, $C, H, S, O, N$ y la ceniza expresan la fracción másica correspondiente a cada elemento bajo los resultados del análisis último de la biomasa en base seca resumido en la Tabla 4 y que corresponden a valores encontrados en la literatura disponible (Se tomaron los valores de la media de los datos como referencia debido a que los porcentajes reportados corresponden en su totalidad a trabajos desarrollados para biomasa vegetal), las diferencias de entalpías a las condiciones alcanzadas para cada componente se muestran en la Tabla 3 y fueron valores tomados de Wark y Richards (1999) y por otro lado, $x, y, z$ corresponden a 1.481, 0.557 y 0.012 respectivamente, calculados con estas mismas fracciones másicas y las masas molares de los elementos de interés para este caso particular y que corresponden al oxígeno $\left(\mathrm{O}_{2}\right)$, nitrógeno $\left(\mathrm{N}_{2}\right)$, dióxido de carbono $\left(\mathrm{CO}_{2}\right)$, hidrógeno $\left(\mathrm{H}_{2}\right)$, Carbono $(\mathrm{C})$ y agua $\left(\mathrm{H}_{2} \mathrm{O}\right)$.

De esta misma manera para la biomasa, La entalpía de formación se obtuvo resolviendo la reacción mostrada en la ecuación (5) con las entalpías de formación de cada componente conocido.

Tabla 4: Análisis último de la biomasa en base seca

\begin{tabular}{|c|c|c|c|c|c|c|c|l|}
\hline \multicolumn{7}{|c|}{ Análisis último (\% base seca) } & \\
\hline & $\mathrm{a}$ & $\mathrm{b}$ & $\mathrm{c}$ & $\mathrm{d}$ & $\mathrm{e}$ & $\mathrm{f}$ & Media & \\
\hline $\mathrm{C}$ & 51.1 & 47.6 & 50.8 & 53.8 & 52.4 & 48.6 & 50.7 & a) Hesas et al. (2013) \\
\hline $\mathrm{H}$ & 7.0 & 5.2 & 6.7 & 7.2 & 6.3 & 5.2 & 6.3 & b) Rashid et al. (2014) \\
\hline $\mathrm{N}$ & 1.9 & 0.2 & 1.3 & 0.0 & 0.6 & 0.1 & 0.7 & c) (Ninduangdee \& Kuprianov (2013) \\
\hline $\mathrm{S}$ & - & - & 0.1 & 0.5 & 0.2 & 0.2 & 0.2 & d) Yan et al. (2005) \\
\hline O & 33.3 & 38.9 & 36.0 & 36.3 & 37.3 & 43.9 & 37.6 & e) Husain et al. (2002) \\
\hline Ceniza & 6.7 & 8.1 & 5.0 & 2.2 & 3.2 & 2.1 & 4.5 & a) Hesas et al. (2013) \\
\hline
\end{tabular}

1 kmol de $\mathrm{CO}_{2} *\left(\overline{h_{f}^{o}}+\bar{h}-\overline{h^{o}}\right)_{\mathrm{CO}_{2}}+\frac{x}{2}$ kmol de $\mathrm{H}_{2} \mathrm{O} *\left(\overline{h_{f}^{o}}+\bar{h}-\overline{h^{o}}\right)_{\mathrm{H}_{2} \mathrm{O}}$

$=1 \mathrm{kmol} d e \mathrm{CH}_{x} \mathrm{O}_{y} \mathrm{~N}_{z} *\left(\overline{h_{f}^{o}}\right)_{C H_{x} O_{y} N_{z}}$

HHV Biomasa $=[349.1 C+1178.3 H+100.5 S-103.4 O-15.1 N-21.1 C e n i z a]_{M_{C H} O y N z}$

$H H V_{\text {Biomasa }}=477487.667 \mathrm{~kJ} / \mathrm{kmol}$

${\overline{h_{f}^{o}}}_{\mathrm{CH}_{x} \mathrm{O}_{y} N_{z}}=-H H V_{\text {Biomasa }}+{\overline{h_{f}^{o}}}_{\mathrm{CO}_{2}}+(y-2) \overline{h_{f_{H_{2}}^{o} \mathrm{O}-\mathrm{l}}}=-458485.7 \mathrm{~kJ} / \mathrm{kmol}$ 
Ahora bien, Considerando las ecuaciones (1) y (2) mostradas con anterioridad para la resolución de la variación de la conductividad del cuesco de palma africana en el reactor y las condiciones de frontera e iniciales mostradas, se muestran ahora las especificaciones de las simulaciones realizadas. Se emplearon entonces, esquemas compactos de diferencias finitas de orden 2, 4 y 6 y la implementación de los métodos de integración de Runge Kutta para $n=1,2$ y 4 respectivamente, utilizando el mismo paso de tiempo (100 segundos) y una grilla de $40 * 40$ para todos los casos.

\section{Sistemas Compactos}

Los métodos de diferencias finitas consisten en una aproximación de las derivadas parciales por expresiones algebraicas con los valores de la variable dependiente en un limitado número de puntos seleccionados. Una de sus desventajas es que a medida que aumenta el orden de la aproximación de la derivada se requiere mayor capacidad computacional.

Para la resolución del problema planteado, se utilizaron esquemas compactos de diferencias finitas de $2^{\text {do }}$ y $6^{\text {to }}$ orden (Lele, 1992; Carpenter et al., 1991; Gao y Sun, 2011). Para ello, teniendo en cuenta que $I_{n} \subseteq I_{m} \subset \square$ tal que $0 \notin I_{m}$, se presenta un esquema compacto para la primera derivada en las ecuaciones (6-10):

$$
u_{i}^{\prime}+\sum_{j \in I_{n}} a_{j} u_{i+j}^{\prime}=\frac{1}{h}\left(b_{0} u_{i}+\sum_{j \in I_{m}} b_{j} u_{i+j}\right)+O\left(h^{p}\right)
$$

La equivalencia de $u_{i+j}^{\prime}$ y $u_{i+j}$ se conoce por la expansión en series de Taylor:

$$
u_{i+j}^{\prime}=\sum_{r=0}^{\infty} \frac{j^{r} h^{r}}{r !} u_{i}^{r+1} \quad u_{i+j}=\sum_{r=0}^{\infty} \frac{j^{r} h^{r}}{r !} u_{i}^{r}
$$

Reemplazando la ecuación (7) en la ecuación (6) se tiene:

$$
u_{i}^{\prime}=\left(b_{0}+\sum_{j \in I_{m}} b_{j}\right) \frac{u_{i}}{h}+\left(-\sum_{j \in I_{n}} a_{j}+\sum_{j \in I_{m}} j b_{j}\right) u_{i}^{\prime}+\sum_{r=2}^{p}\left(\sum_{j \in I_{m}} \frac{j^{r}}{r !} b_{j}-\sum_{j \in I_{n}} \frac{j^{r-1}}{(r-1) !} a_{j}\right) u_{i}^{r} h^{r-1}+O\left(h^{p}\right)
$$

La ecuación (8), es equivalente a las expresiones mostradas por Lele (1992) para aproximar la primera y segunda derivada:

$$
\begin{aligned}
& u^{\prime} \rightarrow \beta f_{i-2}^{\prime}+\alpha f_{i-1}^{\prime}+f_{i}^{\prime}+\alpha f_{i+1}^{\prime}+\beta f_{i+2}^{\prime}=c \frac{f_{i+3}-f_{i-3}}{6 h}+b \frac{f_{i+2}-f_{i-2}}{4 h}+a \frac{f_{i+1}-f_{i-1}}{2 h} \\
& u^{\prime \prime} \rightarrow \beta f_{i-2}^{\prime \prime}+\alpha f_{i-1}^{\prime \prime}+f_{i}^{\prime \prime}+\alpha f_{i+1}^{\prime \prime}+\beta f_{i+2}^{\prime \prime} \\
& =c \frac{f_{i+3}-2 f_{i}+f_{i-3}}{9 h^{2}}+b \frac{f_{i+2}-2 f_{i}+f_{i-2}}{4 h^{2}}+a \frac{f_{i+1}-2 f_{i}+f_{i-1}}{h^{2}}
\end{aligned}
$$

Los valores $I_{m}$ e $I_{n}$ corresponden a los coeficientes de las derivadas de primer y segundo orden en el lado izquierdo y derecho de las ecuaciones (6-10) respectivamente.

Esquemas de Integración

La forma generalizada de los métodos de Runge-Kutta (RK) es la que se muestra a continuación (Hairer, 1993);

$$
y_{i+1}=y_{i}+\phi\left(x_{i}, y_{i}, h\right) h
$$

Donde el incremento se escribe de forma general como: 


$$
\phi=a_{1} k_{1}+a_{2} k_{2}+\ldots+a_{n} k_{n}
$$

Es posible entonces tener varios métodos empleando diferentes números de términos en la función de incremento especificada por $\mathrm{n}$. En el caso de este trabajo se presenta la implementación de los métodos RK para $\mathrm{n}=1,2$ y 4 respectivamente; de esta manera se asumió $\phi=f(t, y)$.

Para el caso donde $n=1$ (Euler) se tiene (Hairer, 1993);

$$
y_{n+1}=y_{n}+f\left(t_{n}, y_{n}\right) h
$$

Para el caso donde $n=2(R K-2)$ se tiene (Ascher y Petzold, 1998);

$$
y_{n+1}=y_{n}+\left(a_{1} k_{1}+a_{2} k_{2}\right) h
$$

Donde;

$$
\begin{aligned}
& k_{1}=f\left(t_{n}, y_{n}\right) \\
& k_{2}=f\left(t_{n}+p_{1} h, y_{n}+q_{11} k_{1} h\right)
\end{aligned}
$$

Para el caso donde $n=4$ (RK-4) se tiene (Ascher y Petzold, 1998);

$$
y_{n+1}=y_{n}+\frac{1}{6}\left(k_{1}+2 k_{2}+2 k_{3}+k_{4}\right) h
$$

Donde;

$$
\begin{aligned}
& k_{1}=f\left(t_{n}, y_{n}\right) \\
& k_{2}=f\left(t_{n}+\frac{h}{2}, y_{n}+\frac{h}{2} k_{1}\right) \\
& k_{3}=f\left(t_{n}+\frac{h}{2}, y_{n}+\frac{h}{2} k_{2}\right) \\
& k_{4}=f\left(t_{n}+h, y_{n}+k_{3}\right)
\end{aligned}
$$

La solución de ecuaciones diferenciales por el método de diferenciación finita con esquemas compactos, se rige por tres pasos fundamentales: a) Establecer qué tipo de esquema utilizar, b) Construir el sistema de ecuaciones correspondiente al esquema elegido, y c) Utilizar un sistema integrador. Retomando la ecuación (9), es necesario encontrar los coeficientes que acompañan cada uno de sus términos, para ello se generaron esquemas compactos para cada uno de los puntos que hacen parte de la malla y un sistema de ecuaciones, de la siguiente forma:

$$
A T^{\prime}=B T \rightarrow T^{\prime}=A^{-1} B T \rightarrow T^{\prime \prime}=\left(A^{-1} B\right)^{2} T
$$

La construcción de las superficies de respuesta que describen la variación de la temperatura de la biomasa con respecto a la posición que ocupa dentro de la tolva, se llevó a cabo utilizando los parámetros de simulación mencionados en la Tabla 5. Dadas las características físicas de la tolva del reactor de lecho fijo analizado, y para simplificar el modelamiento del fenómeno se consideró que su geometría era simétrica. Un rectángulo en este caso, con una condición de borde que restringe el muestreo de datos en base a una configuración binaria, donde el área que no es de interés está representada por ceros, ver la Figura 3. 
Tabla 5: Parámetros utilizados

\begin{tabular}{cccc}
\hline Paso de tiempo & 100 & 1 & 1 \\
\hline $\begin{array}{c}\text { Método de } \\
\text { aproximación }\end{array}$ & $\begin{array}{c}\text { Diferencias finitas por } \\
\text { esquemas compactos } \\
\text { de orden 2 }\end{array}$ & $\begin{array}{c}\text { Diferencias finitas por } \\
\text { esquemas compactos } \\
\text { de orden } 4\end{array}$ & $\begin{array}{c}\text { Diferencias finitas por } \\
\text { esquemas compactos } \\
\text { de orden 6 }\end{array}$ \\
$\begin{array}{c}\text { Método de integración } \\
\text { Número de divisiones }\end{array}$ & $\begin{array}{c}\text { Euler explícito } \\
40\end{array}$ & $\begin{array}{c}\text { Runge Kutta de orden 2 } \\
80\end{array}$ & $\begin{array}{c}\text { Runge Kutta de orden 4 } \\
80\end{array}$ \\
\hline
\end{tabular}

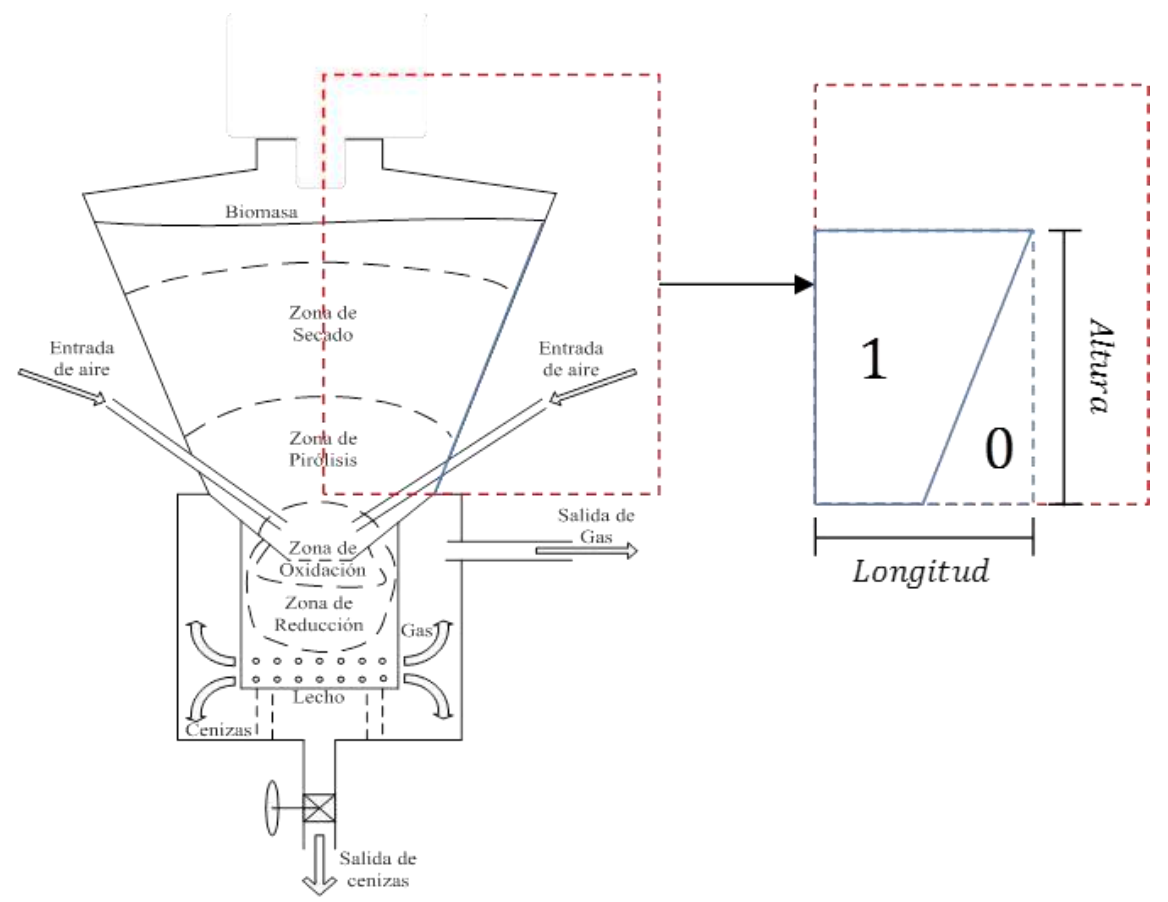

Fig. 3: Esquema de funcionamiento de la máscara

Como criterio de convergencia se escogió comparar las temperaturas obtenidas en las posiciones i-1 e i. Mediante el cálculo de la norma del vector de diferencias de temperaturas, tal como lo expresa la ecuación (23). De esta forma, si el error es menor que la tolerancia (1e-4), el método se detiene y permite visualizar los resultados hacia los cuales converge la temperatura de la biomasa en la tolva.

error $=\left\|\frac{\mid T_{i-1}-T_{i}}{T_{i}} \mid * 100\right\|$

\section{RESULTADOS Y DISCUSIÓN}

La Figura 4 muestra las superficies de respuesta construidas en base a los diferentes métodos mencionados en la Tabla 5. Se observa como al mantener fijos el paso de tiempo y el número de divisiones, los métodos de aproximación e integración influyen significativamente en el comportamiento del tiempo de convergencia de la solución.

Posterior a la aplicación de métodos de aproximación numérica para encontrar el comportamiento de la temperatura de la biomasa en la tolva de un gasificador de lecho fijo, era necesario establecer un criterio de comparación entre simulaciones, que permitiera escoger aquella que aproximándose significativamente a la solución esperada requiera menor tiempo de simulación y capacidad computacional. Para ello, se estableció como criterio medir el error porcentual de los resultados respecto a un punto de referencia, que en este caso es la solución del problema con esquemas compactos de orden $\mathrm{h}^{6}$, sistema integrador Runge-Kutta de orden 4, una malla de $80 \times 80$ y paso de tiempo de 1 segundo. La Figura 5 muestra la superficie de respuesta obtenida bajo la combinación de referencia. Para el cálculo del error porcentual se escogieron 21 puntos de comparación entre ésta solución y las distintas configuraciones propuestas en la Tabla 5. Los 21 puntos se trazaron sobre una línea que va desde $0 \leq \mathrm{y} \leq \mathrm{H}$, todos $\mathrm{x}$-espaciados. 


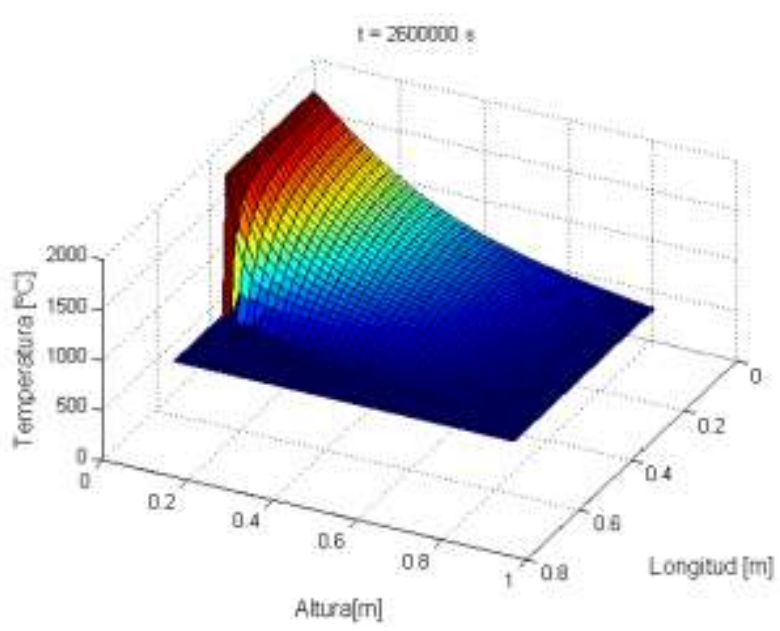

Esquema $\mathrm{h}^{2}-\mathrm{OD} 1$

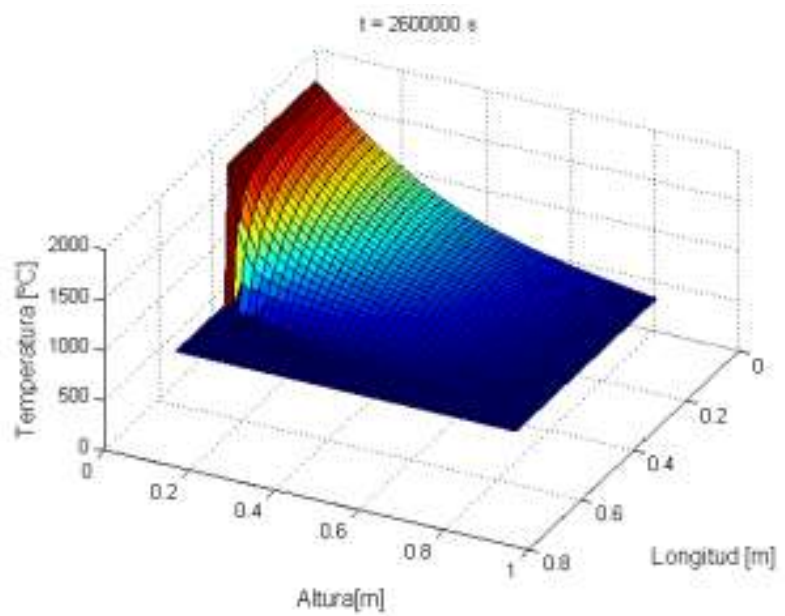

Esquema $\mathrm{h}^{2}-\mathrm{RK} 4$

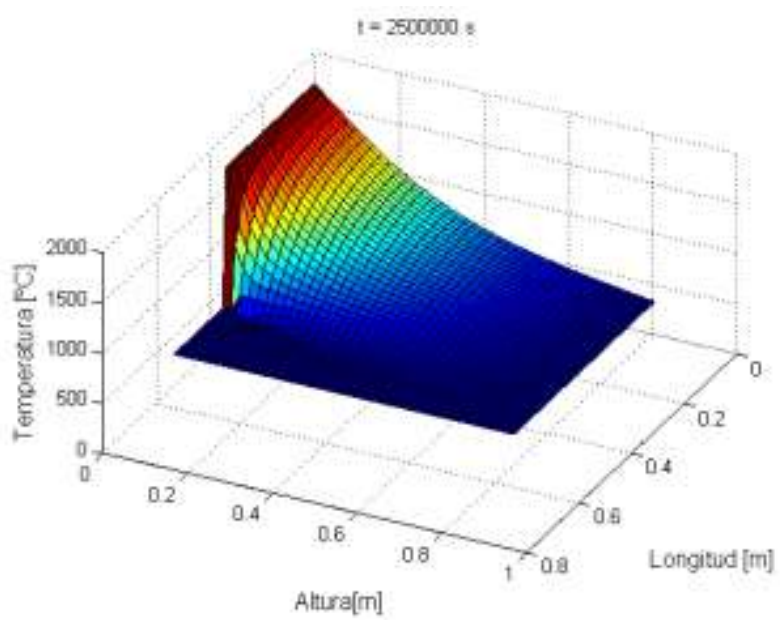

Esquema $h^{4}-\mathrm{OD} 2$

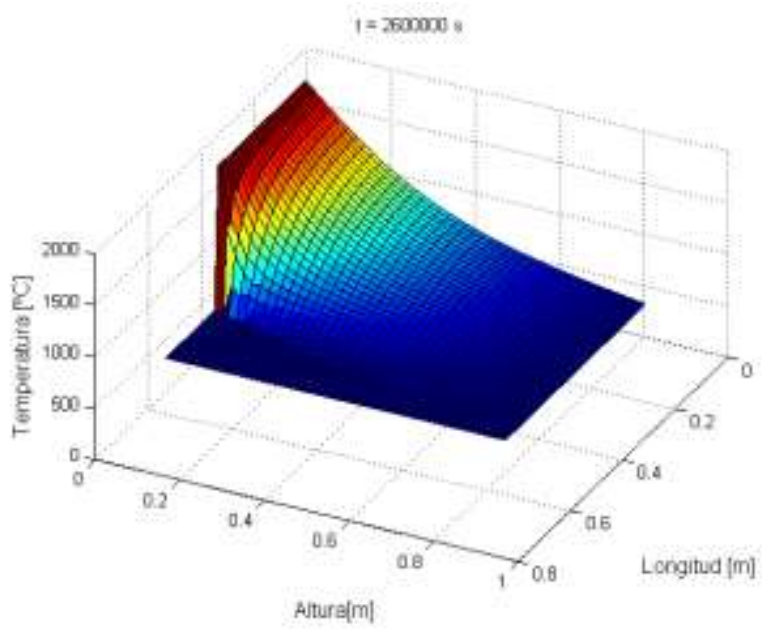

Esquema $\mathrm{h}^{2}-\mathrm{OD} 2$

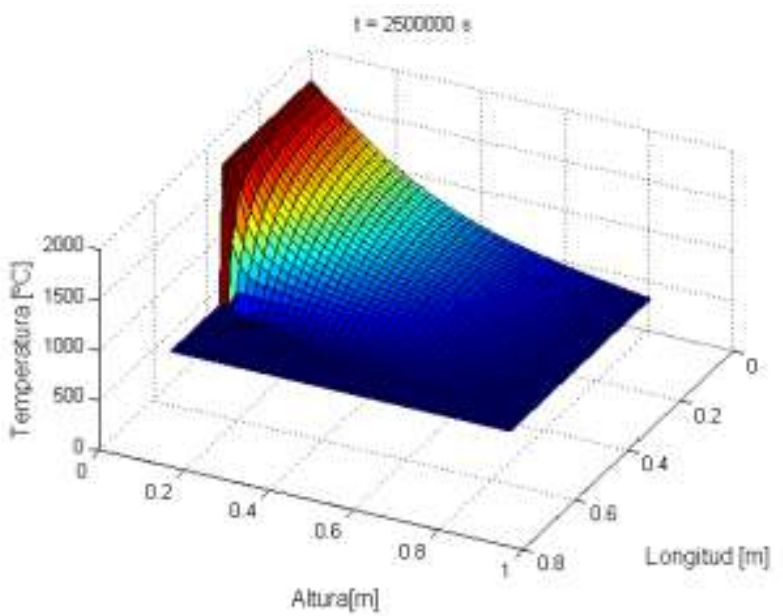

Esquema h4-OD1

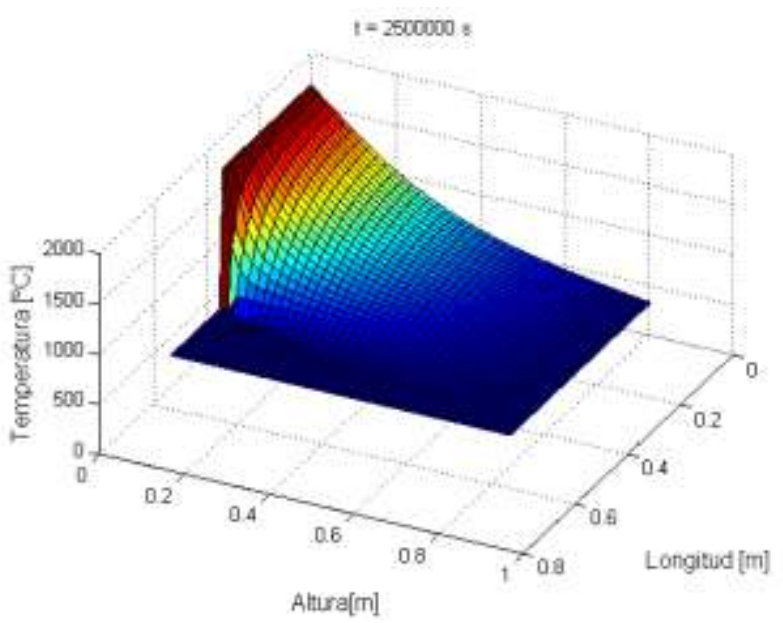

Esquema $\mathrm{h}^{4}-\mathrm{RK} 4$

Fig. 4: Gráficas de superficie de respuesta 


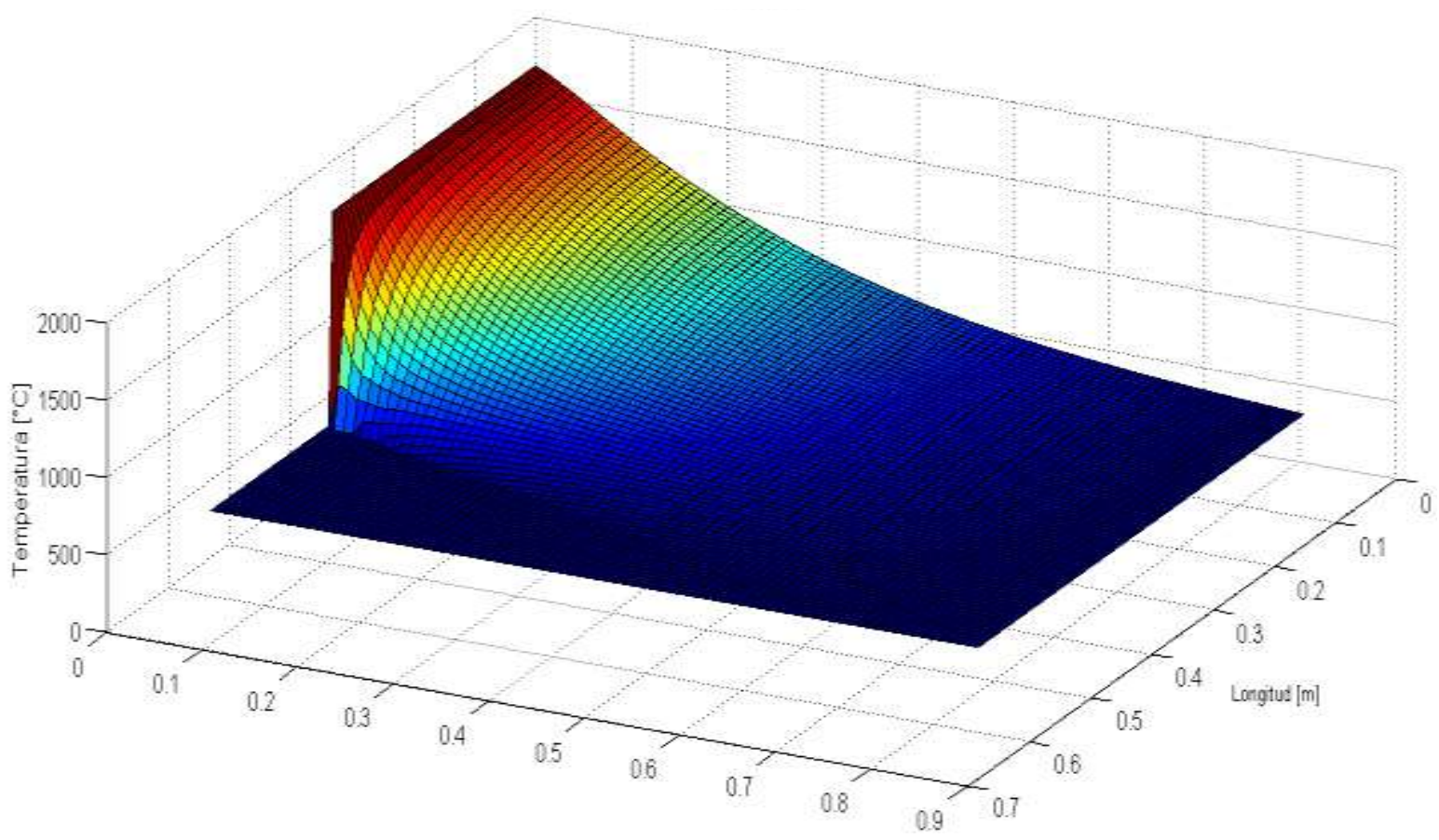

Fig. 5: Esquema $h^{6}-R K 4$

Las Figuras 6 y 7, muestran el comportamiento del error porcentual analizado. La primera compara la totalidad de las combinaciones analizadas, y la segunda corresponde a la comparación de los errores porcentuales cuando se conserva el orden de los sistemas compactos y se varía el método de integración. La tendencia de los errores porcentuales, en la Figura 6, calculados para las aproximaciones obtenidas a partir de esquemas compactos de segundo orden (línea roja) mostraron un comportamiento similar, manejando diferencias entre ellas de aproximadamente $15.3 \mathrm{e}^{-5} \%$.

Por otro lado, con los esquemas de cuarto orden (línea amarilla) mostraron diferencias del orden de $13.6 e^{-5}$ $\%$. Los errores porcentuales más significativos se encuentran a una altura aproximada de $0.5 \mathrm{~m}$ y varían entre $7.22 \%$ y $8.1 \%$, con respecto al punto de referencia. Sobre la altura inicial y final, los errores porcentuales son de $0 \%$ porque bajo estas distancias se establecieron las condiciones de frontera. En la Figura 7, es notable la influencia que tiene el orden de los sistemas compactos sobre la magnitud del error y por ende las aproximaciones realizadas.

Algunos autores han llevado a cabo el análisis de procesos termoquímicos, considerando propiedades variables. Wurzenberg et al. (2002), modelaron este fenómeno, y en su artículo muestran las curvas de temperatura para el mismo. Sin embargo, la aproximación de estos autores sólo considera una partícula. Otros autores (Di Blasi, 2008) muestran que la conductividad térmica de la biomasa presenta un comportamiento anisotrópico, y una de las formas de modelamiento más sencilla, pero a su vez valida, es usar la llamada conductividad térmica efectiva considerada en este artículo, la conductividad varía con la temperatura, más sin embargo no presenta variación en los ejes que conforman la geometría. Como se explicó con anterioridad, uno de los objetivos era evaluar como variaba la conductividad de la biomasa en función de la posición que ocupa en la tolva del gasificador, para ello se resolvió un modelo de combustión teórica con valores de referencia para el sistema y se construyó una correlación entre el perfil de temperaturas calculado y la variación de la conductividad de forma lineal. En la Figura 8, se muestra la correlación entre posición-temperatura y temperatura-conductividad. Los resultados muestran una línea en lugar de una superficie de respuesta, debido a que la verificación de la correlación se realizó para los 21 puntos escogidos en la sección del cálculo de los errores; es decir, los puntos x-espaciados distribuidos sobre una línea que va desde $0 \leq \mathrm{y} \leq \mathrm{H}$.

\section{CONCLUSIONES}

Los resultados permiten observar que existe mejor ajuste y resolución del problema cuando se trabaja con sistemas integradores de orden superior, así como también que tanto la variación del orden de los esquemas compactos de diferencias finitas como la variación del método de integración son los factores más significativos en la búsqueda de mayor grado de aproximación y resolución de la variable analizada. El paso de tiempo y la malla resultan de mayor peso en la capacidad computacional requerida (Es decir, mayor tiempo empleado por el equipo para resolver el fenómeno). 


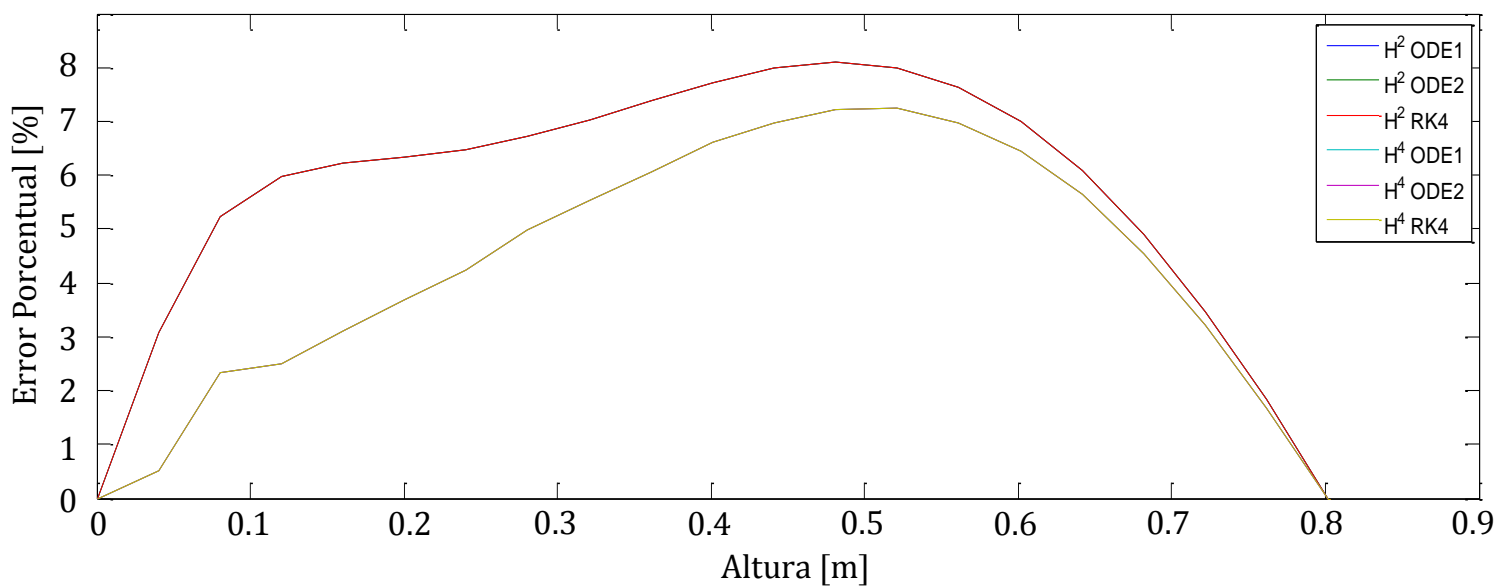

Fig. 6: Error porcentual entre los sistemas analizados
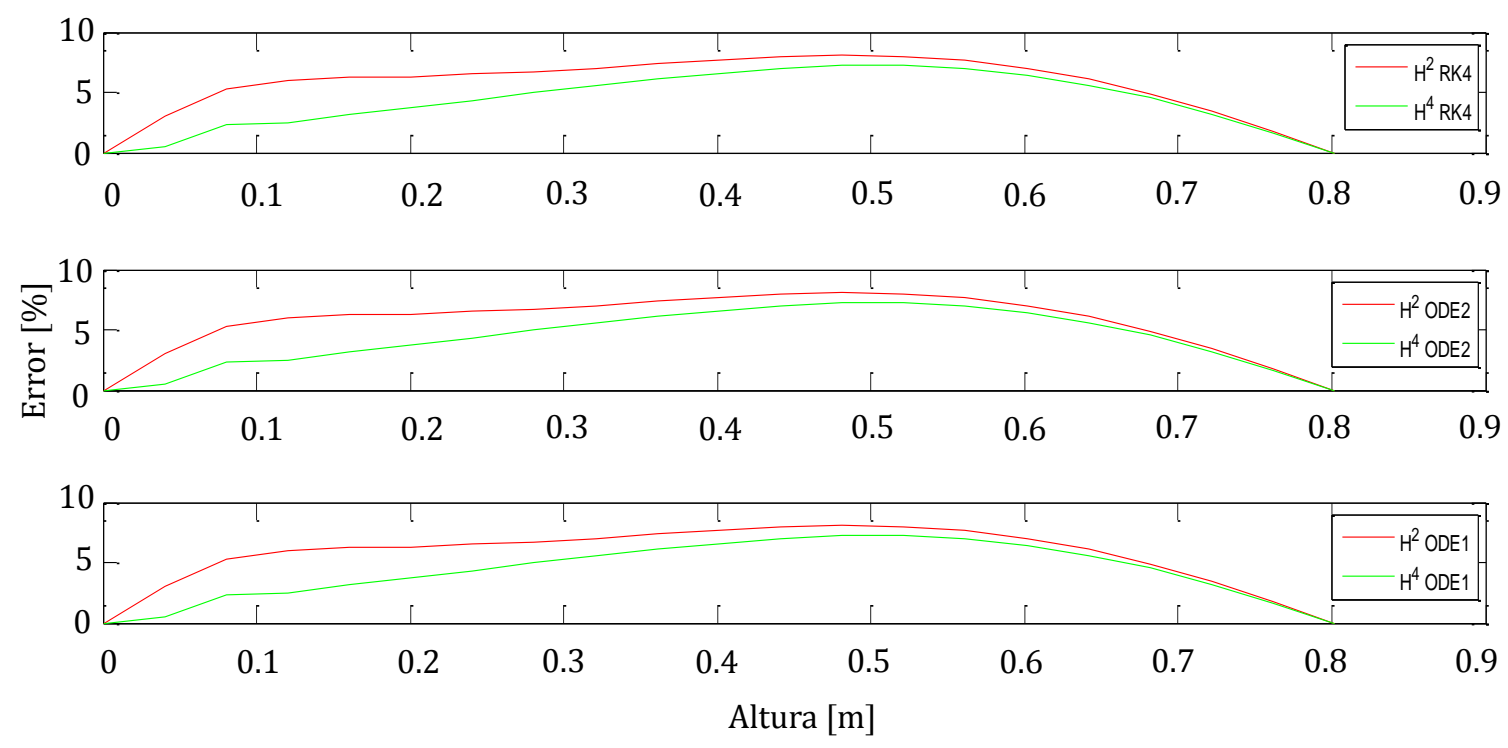

Fig. 7: Error porcentual por corridas analizadas

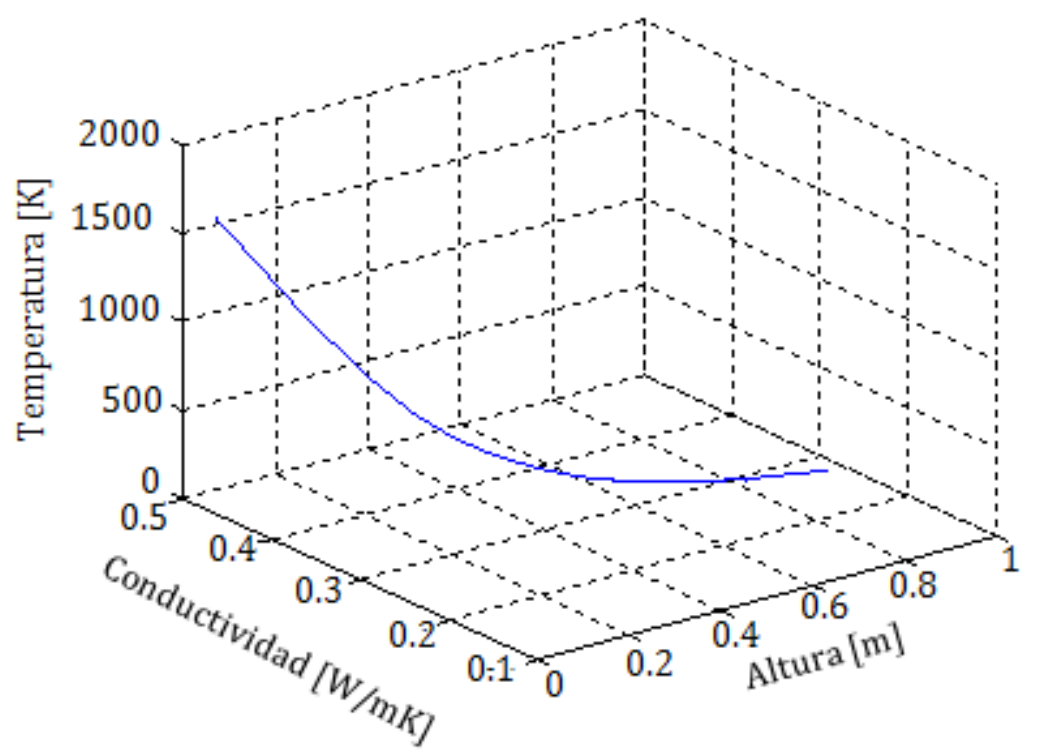

Fig. 8: Correlación establecida entre la posición, temperatura y la conductividad 
En este orden, la aproximación obtenida bajo el esquema compacto de orden 4 y el método de integración Runge Kutta de cuarto orden, fué la más cercana al punto de referencia analizado correspondiente a una resolución con esquemas compactos de orden $\mathrm{h}^{6}$, sistema integrador Runge-Kutta de orden 4, una malla de $80 \times 80$ y paso de tiempo de 1 segundo, mostrando diferencias del orden de $13.6 e^{-5} \%$ y los errores porcentuales más significativos entre $7.22 \%$ y $8.1 \%$ a una altura aproximada de $0.5 \mathrm{~m}$ medida desde el fondo de la tolva en la dirección ascendente.

Respecto a la variación de la conductividad del material, se observó que esta variable aumenta a medida que se incrementa la temperatura, es decir, es inversamente proporcional a la distancia (posición) medida desde la base menor de la tolva en la dirección analizada, es decir, a medida que se avanza en la dirección +Y, la conductividad del material disminuye. Los resultados muestran una línea en lugar de una superficie de respuesta, debido a que la verificación de la correlación se realizó para los 21 puntos escogidos en la sección del cálculo de los errores ( $\mathrm{x}$-espaciados distribuidos sobre una línea que va desde $0 \leq \mathrm{y} \leq \mathrm{H}$ )

\section{AGRADECIMIENTOS}

Los autores agradecen al Departamento del Atlántico por el apoyo a través de recursos del Sistema General de Regalías - Fondo de Ciencia, Tecnología e Innovación.

\section{REFERENCIAS}

Abd Rashid, R., Mohd Salleh, H., Hanafi Ani, M., Azhani Yunus, N., Akiyama, T. y Purwanto, H., Reduction of low grade iron ore pellet using palm kernel shell, Renewable Energy, 63, 617-623 (2014)

Abnisa, F., Wan Daud, W., Husin, W. y Sahu, J., Utilization possibilities of palm shell as a source of biomass energy in Malaysia by producing bio-oil in pyrolysis process, Biomass \& Bioenergy, 35(5), 1863-1872 (2011)

Ahmad, A. A., Zawawi, N. A., Kasim, F. H., Inayat, A. y Khasri, A., Assessing the gasification performance of biomass: A review on biomass gasification process conditions, optimization and economic evaluation, Renewable and Sustainable Energy Reviews, 53, 1333-1347 (2016)

Ahumada, L., Verdeza, A., Bula, A. y Lombana, J., Optimización de las Condiciones de Operación de la Micro-gasificación de Biomasa para Producción de Gas de Síntesis, Inf. Tecnol., 27(3), 179-188 (2016)

Ascher, Uri M. y Petzold, Linda Ruth., Computer methods for ordinary differential equations and differentialalgebraic equations, 1를 Ed., Philadelphia, USA, SIAM, ISBN 0898714125 (1998)

Basu, P., Biomass Gasification and Pyrolysis: Practical Design and Theory, Burlington, USA, Elsevier (2010)

Channiwala, S. y Parikh, P., A unified correlation for estimating HHV of solid, liquid and gaseous fuels, Fuel, 81(8), 1051-106 (2002)

Chen, C., Horio, M. y Kojima, T., Use of numerical modeling in the design and scale-up of entrained flow coal gasifiers, Fuel, 80(10), 1513-1523 (2001)

Chen, C., Masayuki, H. y Kojima, T., Numerical simulation of entrained flow coal gasifiers. Part I: modeling of coal gasification in an entrained flow gasifier, Chemical Engineering Science, 55(18), 3861-3874 (2000)

Di Blasi, C., Modeling chemical and physical processes of wood and biomass pyrolysis, Progress in Energy and Combustion Science, 34(1), 47-90 (2008)

$\mathrm{Di}, \mathrm{Z}$. y Weizhong D., Accurate finite difference schemes for solving a 3D micro heat transfer model in an Ncarrier system with the Neumann boundary condition in spherical coordinates, Journal of Computational and Applied Mathematics, 235, 850-869 (2010)

García-Ubaque, C., Vaca-Bohórquez, M. y Talero, G., Aprovechamiento de Biomasa Peletizada en el Sector Ladrillero en Bogotá-Colombia: Análisis Energético y Ambiental, Inf. Tecnol. 24(3), 115-120 (2013)

Gupta, Murlidhar, Yang, Jin y Roy, Christian, Specific heat and thermal conductivity of softwood bark and softwood char particles, Fuel 82, 919-927 (2003)

Hairer, Ernst., Wanner, Gerhard y Nørsett, Syvert P., Solving Ordinary Differential Equations I, Nonstiff Problems, ISBN: 978-3-540-56670-0, Springer Series in Computational Mathematics, Vol. 8 (1993)

Harada, T., Hata, T. y Ishihara, S., Thermal constants of wood during the heating process measured with the laser flash method, J. Wood Science (44), 425-431 (1998)

Hoseinzadeh Hesas, R., Arami-Niya, A., Ashri Wan Daud, W. y Sahu, J., Comparison of oil palm shell-based activated carbons produced by microwave and conventional heating methods using zinc chloride activation, Journal of Analytical and Applied Pyrolysis, 104,176-184 (2013) 
Husain, Z., Zainac, Z. y Abdullah, Z., Briquetting of palm fibre and shell from the processing of palm nuts to palm oil, Biomass and Bioenergy, 22(6), 505-509 (2002)

Ibarra, María del Carmen., La ecuación de calor de Fourier: resolución mediante métodos de análisis en variable real y en variable compleja, UTN Facultad Regional Resistencia: II Jornadas de Investigación en Ingeniería del NEA y Países Limítrofes, Consultado el 10 de febrero de 2017, p. 3 (2012)

Kumar, A., Jones, D. y Hanna, M., Thermochemical Biomass Gasification: A Review of the Current Status of the Technology, Energies, 556-581 (2009)

Lele, S., Compact finite difference schemes with spectral-like resolution, J. Comput. Phys (103), 16-42 (1992)

Mehdi S, S., Haghighi, M. y Rahmani, F., Syngas production from co2-reforming of ch4 over sol-gel synthesized ni-co/al2o3-mgo-zro2 nanocatalyst: effect of zro2 precursor on catalyst properties and performance, Química Nova, 38(4) (2015)

Melgar, A., Perez, J. y Horrillo, A., Biomass gasification process in a downdraft fixed bed gasifier: a real time diagnosis model based on gas composition analysis, Revista Facultad de Ingeniería Universidad de Antioquia(49) (2009)

Mendoza, J., Bula, A., Gómez, R. y Corredor, L., Análisis Exergético de la Gasificación de Biomasa, Información Tecnológica, 23(5), 85-96 (2012)

Moreno, A, L. S., Rodriguez C, F. E., Afanador R, L. E., y Grosso V, J. L., Syngas obtainment from the gasification of asphaltenes of the san fernando crude oil, Ciencia, Tecnología y Futuro, 3(5), 189-204 (2009)

Moura Pinto, Edna., Regobello, Ronaldo., Munaiar Neto, Jorge y Calil Junior, Carlito., Estudio TeóricoExperimental sobre la degradación térmica de vigas de Eucalyptus, Maderas, Cienc. Tecnol, (2), 135-144 (2007)

Ninduangdee, P. y Kuprianov, V., Study on burning oil palm kernel shell in a conical fluidized-bed combustor using alumina as the bed material, Journal of the Taiwan Institute of Chemical Engineers, 44(6), 1045-1053 (2013)

Oviedo, J., Casas, A., Valencia, J. y Zapata, J., Análisis de la Medición de la Biomasa en Fermentación en Estado Sólido empleando el Modelo Logístico y Redes Neuronales, Información Tecnológica, 25(4), 141152 (2014)

Perez, J., Borge, D. y Agudelo, J., Proceso de gasificación de biomasa: una revisión de estudios teóricoexperimentales, Revista Facultad de Ingeniería Universidad de Antioquia (52), 95-107 (2010)

Qingluan, X. y Rodney, O. F., Computational Modeling of Biomass Thermochemical Conversion in Fluidized Beds: Particle Density Variation and Size Distribution, Ind. Eng. Chem. Res, 54(16), 4084-4094 (2015)

Sordi, A., Da Silva, E., Neto, A., Lopes, D., Pinto, C. y Araújo, P., Thermodynamic simulation of biomass gas steam reforming for a solid oxide fuel cell (SOFC) system, Brazilian Journal of Chemical Engineering, 26(4), 745-755 (2009)

T.V.S, Sekhar, B. Hema Sundar Raju y P.V.S.N, Murthy, Higher order compact scheme for laminar natural convective heat transfer from a sphere, Applied Mathematical Modelling, 40, 2039-2055 (2016)

Wark, Kenneth y Richards, Donald, Thermodynamics, 6 $6^{\text {th }}$ Ed., $1664 \mathrm{pp}$, WCB/McGraw-Hill, ISBN 0-07068305-0 (1999)

Wurzenberger, J. C., Wallner, S., Raupenstrauch, H., y Khinast, J. G., Thermal conversion of biomass: Comprehensive reactor and particle modeling, AIChE Journal, 48(10), 2398-2411 (2002)

Yan, R., Yang, H., Chin, T., Tee Liang, D., Chen, H. y Zheng, C., Influence of temperature on the distribution of gaseous products from pyrolyzing palm oil wastes, Combustion and Flame, 142, 24-32 (2005)

Yepez M, D. M. y Chejne J, F., Gasificación de biomasa residual en el sector, Revista ION, 25(2) (2012) 